\title{
II. Upon a photo-chemical method for the determination of organic matter in potable water
}

\section{Dr. Albert R. Leeds}

To cite this article: Dr. Albert R. Leeds (1883) II. Upon a photo-chemical method for the determination of organic matter in potable water , Philosophical Magazine Series 5, 16:97, 9-13, DOI: $10.1080 / 14786448308627387$

To link to this article: http://dx.doi.org/10.1080/14786448308627387

曲 Published online: 28 Apr 2009.

Submit your article to this journal $[\pi$

Џ Article views: 2

Q View related articles 
a periodic variation of the electric forces within the insulating fluid between the electrodes.

The displacement in the direction of a diminution of the refraction-index corresponds to a raising of the temperature of the insulating fluid from $0^{\circ} \cdot 0001$ to $0^{\circ} \cdot 1 \mathrm{C}$.; it increases with the difference of electric potentials of the electrodes and the viscosity of the fluid, and may be owing to a heating of the fluid by friction, since the fluid-particles are moved to and fro and stirred round by the electric forces between the electrodes.

The increase of the refraction-index occurs, in some fluids, only at the commencement of the electric action ; in others it alternates with a diminution at short intervals during the entire continuance of the electric action.

II. Upon a Photo-chemical Method for the Determination of Organic Matter in Potable Water. By Dr. AlberT R. LEEDs*.

WITHOUT attempting to revive at this moment the discussion as to the sources of error inherent in the present methods employed for the estimation of organic matter in potable waters, or as to the relative value of the inferences drawn from the results arrived at by the various analytical methods, I shall proceed to state the outlines of the new process, and the experiments thus far made to test its accuracy and applicability. The process is founded on the fact of the ready decomposability of certain salts of silver when exposed, in the presence of organic matter and in the state of solution, to the action of light. As a preliminary step it was needful to determine whether these salts were likewise reduced, even when organic matter was not present; and to this end the following experiments were tried :-

1. To 100 cubic centim. of ammonia-free distilled water, 5 cubic centim. of a decinormal solution of nitrate of silver was added, and the liquid exposed to the sunlight for 48 hours in a well-stoppered comparison-tube.

2. The same, but after addition of ammonia just sufficient to redissolve the precipitate first formed.

3. Same as 1, but the silver first precipitated as chloride, and then redissolved by ammonia.

4. Same as 1, but the silver precipitated as cyanide, and then redissolved in cyanide of potassium.

5. Same as 1 , but the precipitate first formed with sodium hyposulphite just redissolved in excess of reagent.

* Communicated by the Author. 
After two days' exposure, no precipitation of reduced silver occurred in the neutral solution of argentic nitrate, nor in that of the ammonio-argentic oxide, nor in the ammonio-argentic chloride, nor in the sodio-argentic hyposulphite.

Having thus established the fact that no reduction of silver occurs in case organic matter is rigidily excluded, even after prolonged exposure to sunlight, the next point of inquiry was to determine which of these five solutions was most affected by such organic matters as are ordinarily present in potable water. The inquiry as to the relative energy of action of different kinds of organic matter in general did not necessarily form a part of the present investigation.

In the study of this point, a sample of potable water was made use of from the river Schuylkill, the water-supply of the city of Philadelphia. This drinking-water had been affected by a sudden cachexy in the month of January, becoming most offensive to taste and smell; and the authorities of the city had requested me to investigate the causes of its nonpotability. The scope of the inquiry being thus narrowed to the finding of specific causes, I was necessitated to tax the capabilities of the methods at present possessed by water-analysts, and was furthermore led to test this new one. Now on adding, to the five silver solutions prepared as in the first series of experiments, 100 cubic centim. of this Schuylkill water, it was found, after 5 hours' exposure to sunlight, that the ammonio-argentic oxide was slightly changed, the potassioargentic cyanide was unaffected. Of the remaining three, the sodio-argentic hyposulphite threw down the heaviest precipitate, the ammonio-argentic chloride a precipitate nearly as great, and the neutral solution of argentic nitrate the least.

From these experiments it would appear that the solution of argentic hyposulphite in excess of sodium hyposulphite is the reagent best adapted for use in this actinic method. But subsequent experiments showed that the neutral solution of argentic nitrate fulfilled all needed requirements, and that the labour of preparing a special reagent was uncalled for. The method finally adopted is as follows:-

250 cubic centim. of the natural water is treated with 10 cubic centim. of decinormal silver solution in the tall glassstoppered cylinders or bottles. The waters become turbid and frequently coloured; but after a moderate interval, usually less than two days, the turbidity entirely disappears and the entire precipitate collects at the bottom of the vessel. In case sufficient silver has been employed (and the amount recommended is ordinarily a large excess), this clarification indicates 
an end-reaction, and it is useless to expect a renewed precipitation in case the filtrate be submitted to second exposure. The precipitates are collected on asbestos filters, similar to those used in the determination of carbon in steel, washed first with water, then with strong ammonia to redissolve coprecipitated chloride, dissolved in nitric acid, evaporated to dryness, redissolved in nitric acid, and the silver determined by Pisani's method. All these washings are but the work of a few minutes with the aid of a water-pump. The motallic silver may be weighed directly ; but the method indicated is more rapid and equally accurate.

Six samples of the Philadelphia water (Nos. 230-235) were so treated; and duplicate determinations of the oxygen required to oxidize the organic matters were made by potassium permanganate. Finding that the results better accorded with the information imparted by the other data of water-analysis than those obtained by the permanganate, the method was systematically applied to the examination of the Passaic water (water-supply of Newark and Jersey City).

The results were as follows:-

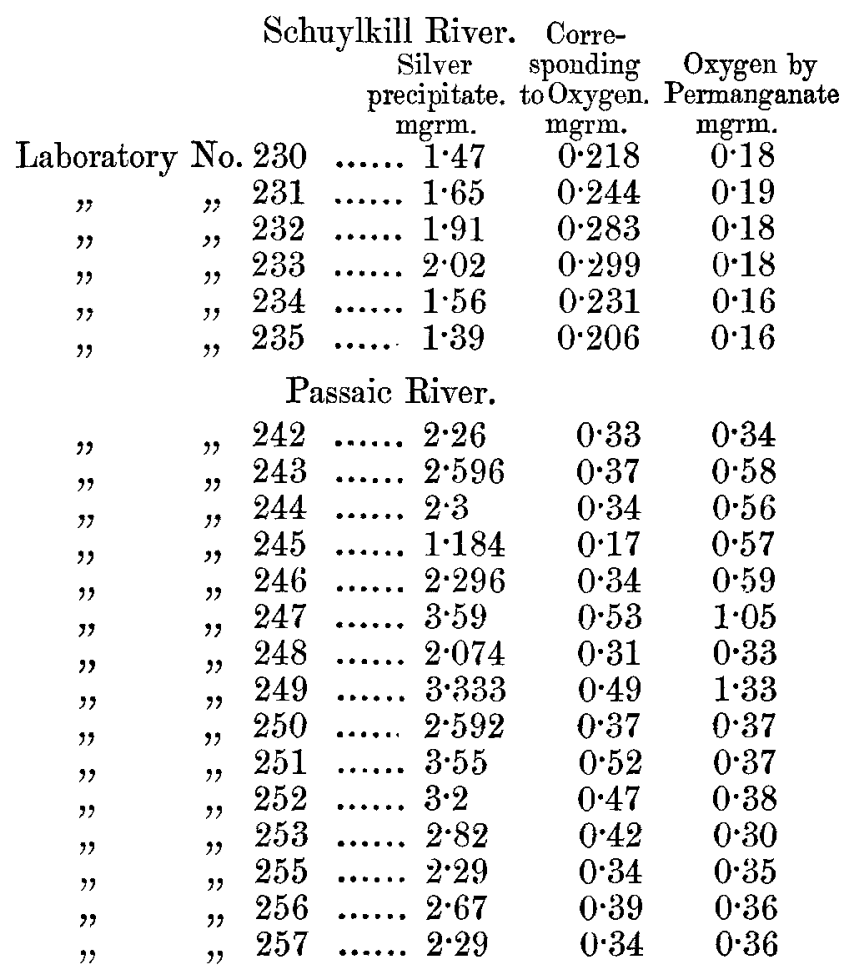


It will be noted that whilst there is a close agreement in many cases between the results obtained by these utterly diverse methods, yet in others there is a striking difference-the higher result being uniformly obtained by the actinic method with the Schuylkill waters, but generally by the permanganate with the Passaic waters. Now investigation revealed that the non-potability of the former was connected with a state of retarded or arrested oxidation, the ice-covered stream having a striking deficiency of oxygen as compared with its normal percentage, and being laden with products of organic decomposition. In the Passaic, besides the sewage there is a great volume of trade pollution; and it is in connexion with such samples (like Nos. 247 and 249, which show the points where the refuse of the great manufacturing city of Paterson is discharged into the Passaic) that the discrepancy is greatest, and the oxygen as determined by the permanganate most exceeds the amount as determined by reduction of silver. The general inference from these experiments was that, while potassium permanganate at the boiling-point (the determinations were made at $98^{\circ} \mathrm{C}$.) was decomposed to a greater or less extent by most organic substances soluble in water, the reduction of the silver was to be ascribed to easily decomposable organic bodies akin in their nature to sewage.

To test this point some weak hay-infusion and highly dilute urine were added to two portions of silver solution and exposed to sunlight. A dense mirror quickly formed on sides and bottom of vessels. Solutions of cane-sugar, moist sugar, and clear starch were added. No darkening of the liquid occurred in the first two cases, and after a short while the slight grey precipitate ceased to increase. This taint action was not due to the sugar, but to a minute amount of foreign organic matter contained in it. Starch produced no change, neither did nitrobenzol. Urea caused a scarcely perceptible precipitate. The powerful base aniline decomposed the silver salt, forming aniline nitrate; and afterwards the liberated argentic oxide was decomposed, its silver forming a beautiful mirror and the solution becoming dyed with rosaniline. This reduction by organic bases might indeed be employed for the silvering of mirrors and the interior of glass ornaments. But such bodies would be rarely present, even in trade-polluted streams; and if they were, would be readily detected, and their influence allowed for.

Of course, the reduction of ammoniacal solution of silver in presence of alcohol, naphtha, and essential oils like cassia, caraway, cloves, \&c., will occur to the reader; so also will the reduction of the alkalized silver liquid by alcoholic solu- 
tion of grape-sugar, or of milk- or cane-sugar, or by aldehydes \&c. But the reductions thus effected bear little analogy to that occurring in a neutral solution, otherwise permanent, under the infiuence of light. In conclusion, I desire to add that this publication is in one sense premature; for while the process has been constantly employed in my own laboratory during the past four months, I have not as yet had the opportunity to apply it to so great a variety of waters as I had intended. But finding that various chemists to whom I have communicated it have always adopted the method, I publish it in order to obtain the benefit of wider and more severe criticism.

\section{On Mr. Ferrel's Theory of Atmospheric Currents. By D. D. HeATH, Esq.*}

T $\mathrm{T}$ has recently come to my knowledge that teachers of 1 Physical Science who have not had a mathematical training (and the specialism of the day must be increasing the class) trust to Mr. Ferrel, of Cambridge in the United States, as a sound and accepted authority for dynamical principles and conclusions relating to atmospheric currents; and, not to quote other confirmations of this fact from communications to 'Nature' and elsewhere, I find that Dr. Haughton, of Dublin, thus speaks of him in some lectures on Physical Geography (1880):-

"We are not able, in the present state of our knowledge, to form a complete mathematical theory of atmospheric currents caused by the unequal expansion of the air by solar heat. ... But we are able to satisfy the equations of motion by a special arrangement of all the atmospheric currents and barometric pressures, which gives us a solution of the problem that explains every important fact... This solution is due to $\mathrm{Mr}$. W. Ferrel, of the United-States Coast Survey, 'The Motions of Fluids and Solids relative to the Earth's Surface' (New York, 1860)."

This is a cautiously worded passage; and I suspect Dr. Haughton has not examined very carefully the connexion between Mr. Ferrel's premises and conclusions. I only quote it as a justification for my thinking it must be worth while to show the unsoundness of his work and the baselessness of his conclusions, if I am right in the estimate I have made of them. I may as well premise, however, that I possess no familiarity with the facts of meteorology, on which Mr. Ferrel may have every claim to speak; I only address myself to his principles and methods of proof.

* Communicated by the Author. 